\title{
Design, Synthesis and In-Silico Studies of Novel Chalcones as Anti-Prostate Cancer and Cathepsin B Inhibitors
}

\author{
Dalia Hussein Soliman ${ }^{1}$, Amel Mostafa Farrag ${ }^{1}, \mathrm{Ola} \mathrm{Omran}^{2}$ \\ ${ }^{1}$ Department of Pharmaceutical Chemistry, Faculty of Pharmacy, Al-Azhar University, Cairo, Egypt. \\ ${ }^{2}$ Department of Radiation Biology, Natural Center for Radiation Research and Technology, Atomic Energy Authority, Cairo, Egypt.
}

\begin{tabular}{|c|c|}
\hline ARTICLE INFO & ABSTRACT \\
\hline Article history: & \multirow{6}{*}{$\begin{array}{l}\text { In the present study a series of novel } N \text { - }(4-3 \text {-(pheny)- } 1 \text {-prop-2-en-1-one phenyl) benzamide derivatives (5a-p) } \\
\text { were synthesized, characterized and evaluated for their anti-prostate cancer activities against PC-3 prostate } \\
\text { adenocarcinoma cell line using the SRB method. The cathepsin B inhibition potential was further tested by the } \\
\text { using enzyme-linked immunosorbent assay. The furyl derivative } \mathbf{5 p} \text { was the most active candidate, (IC50 = } \\
5.597 \mu \mathrm{M}) \text {, against prostate cancer cell line, PC-3. This derivative also demonstrated } 50.4 \% \text { reduction in } \\
\text { concentration of cathepsin B, additionally, compounds } \mathbf{5 b} \text { and } \mathbf{5 m} \text { showed } 53.2 \text { and } 56.6 \% \text { reduction in } \\
\text { concentration of cathepsin B, respectively. In conclusion, compounds } \mathbf{5 b}, \mathbf{5 m} \text { and } \mathbf{5 p} \text { showed good activity both } \\
\text { as antiproliferative and as inhibitors of Cathepsin B production. Moreover, a pharmacophore model was } \\
\text { constructed, a QSAR study was carried out where a model was successfully built from which the } \\
\text { physicochemical parameters were correlated to the activity. This developed QSAR model was found statistically } \\
\text { significant and had good predictive power. Furthermore, molecular docking studies of the active derivatives } \\
\text { were also carried out, where they were found to maintain the essential key interactions, specially with the } \\
\text { cysteine residue, Cys } 29 \text {. }\end{array}$} \\
\hline Received on: 27/11/2016 & \\
\hline ted on: $22 / 01 / 2017$ & \\
\hline Available online: $30 / 07 / 2017$ & \\
\hline Key words: & \\
\hline $\begin{array}{l}\text { Prostate cancer; cathepsin B; } \\
\text { chalcones. }\end{array}$ & \\
\hline
\end{tabular}

\section{INTRODUCTION}

Prostate cancer is one of the most frequently diagnosed malignancies in men. High mortality rate in patients could be mainly attributed to the invasiveness and metastasis in the prostate cancer progression. Generally, malignant cells show an increased rate of proteolytic activity, thus resulting in enhanced invasion and metastasis (Heidenreich et al., 2008; Fidler 1990). Many proteolytic enzymes are involved in the degradation of the extracellular matrices. One of them is cathepsin B, a lysosomal cysteine protease (Tanaka et al., 2000). Interestingly, this enzyme proved to be a prognostic marker for many cancers (Koblinski et al., 2000; Nouh et al., 2011). The overexpression of cathepsin B has been observed in malignant tumors and has been implicated in the migration and invasion of tumors (Agrawal and Soloane 2014; Fernandez et al., 2001; Mohanam et al., 2001; Olson and Joyce 2015).

\footnotetext{
* Corresponding Author

Email: odihss3 @ gmail.com
}

Aberrant regulation of cathepsin B could lead to increased degradation of the extracellular matrix, and thereby might attribute to the infiltrative nature of the tumor cells (Ruan et al., 2015), simultaneously down-regulation of cathepsin B showed the opposite effects (Ruan et al., 2016). In prostate cancer, cathepsin B plays a crucial role in its invasion and metastasis (Sinha et al., 2001; Miyake 2004; Nalla et al., 2010). Consequently, targeting molecules involved in metastasis could be considered an effective route for treatment of prostate cancer. Thereby, the search for selective cathepsin B inhibitors has been considered an active area of research, because its inhibition reduces both tumor cell motility and invasion. However, to date, none of these inhibitors have been clinically approved. Chalcone derivatives have proven to be potential scaffolds in drug discovery due to their enhanced biological activities as well as their safety profiles (Syam et al., 2012). Moreover, many chalcones have also emerged as effective anticancer agents against most human malignancies (Overmeyer et al., 2011; Shah 2008; Boumendjel et al., 2009; Katsori and Hadjipavlou-Latina 2009). 
They inhibit tumor growth and cause apoptosis in prostate cancer cells (Zhou et al., 2013; Nelson et al., 2009; Fu et al., 2006; Yun et al., 2008; Desmulle et al., 2006; Szliszka et al., 2010). Furthermore, chalcones in particular have been reported to exhibit significant cathepsin B inhibition (Lee et al., 2013; Kim et al., 2014; Shewata and Neera, 2015). The aforementioned data, encouraged us to synthesize novel derivatives of $\mathrm{N}$-(4-3-(pheny)1-prop-2-en-1-one phenyl) benzamide derivatives (5a-p) and evaluate their anti-prostate cancer activities as well as their cathepsin B inhibition potential.

Additionally, a pharmacophore model was constructed to identify some of the structural requirements essential for the activity. Moreover, a 2D QSAR model, for the novel compounds, was generated and validated with the aim of identifying the molecular descriptors controlling the activities of these agents. Finally, molecular docking studies were also carried out to determine the binding mode of the active compounds inside the active site of cathepsin B enzyme.

\section{MATERIALS AND METHODS}

\section{CHEMISTRY}

The solvents used were commercially available and distilled before use. Reactions were monitored by thin-layer chromatography (TLC) on silica gel plates (60 F254), visualizing with ultraviolet. Infrared spectra $(\mathrm{KBr})$ were recorded on FT-IR 5300 spectrophotometer and Perkin Elmer spectrum RXIFT-IR system $(v, \mathrm{~cm}-1) .{ }^{1} \mathrm{HNMR}$ spectra were recorded on Varian Gemini spectrophotometer $(300 \mathrm{MHz})$ in DMSO-d6 as solvent. The proton chemical shifts $(\delta)$ are relative to tetramethylsilane (TMS, $d=0.00$ ) as internal standard and expressed in ppm. Spin multiplicities are given as $\mathrm{s}$ (singlet), $\mathrm{d}$ (doublet), $\mathrm{t}$ (triplet), and $\mathrm{m}$ (multiplet) as well as b (broad). Coupling constants (J) are given in hertz.

Melting points were determined by using melting point apparatus and are uncorrected. Mass spectra were obtained on a GC Ms-QP 1000 EX mass spectrometer at $70 \mathrm{eV}$. Microanalyses were performed using a $\mathrm{C} \mathrm{H} \mathrm{N} \mathrm{S/O} \mathrm{analyzer.} \mathrm{Elemental} \mathrm{data} \mathrm{are}$ within $\pm 0.4 \%$ of the theoretical values. All yields reported are notoptimized. The chemical reagents used in synthesis were purchased from Fluka, Sigma and Aldrich.

\section{$N$-(4-Acetylphenyl)-4-chlorobenzamide (3a)}

To a solution of p-aminoacetophenone $(0.01 \mathrm{~mol})$ in dimethyl formamide $(20 \mathrm{~mL})$ an equimolar amount of 4-chloro benzoyl chloride $(0.01 \mathrm{~mol})$ was added. The mixture was stirred at $0^{\circ} \mathrm{C}$ for $3 \mathrm{~h}$, after which crushed ice was added with continuous stirring. A heavy precipitate was obtained, which was filtered, washed with water and after drying it was crystallized out from ethanol. The yield was $60 \%$ and the melting temperature was 160 $162^{\circ} \mathrm{C}$. The IR spectrum, $(\mathrm{KBr}): v_{\max }, \mathrm{cm}^{-1}$, was: $3333(\mathrm{NH}), 1649$ (br., $2 \mathrm{C}=\mathrm{O}) ;{ }^{1} \mathrm{H}$ NMR $(300 \mathrm{MHz}$, DMSO-d6) $\delta$ (ppm) : $2.55(3 \mathrm{H}$; s, CH3), 7.55 ( $2 \mathrm{H} ; \mathrm{d}, \mathrm{J}=9 \mathrm{~Hz}, \mathrm{AB}$ system, ), 7.61 ( $2 \mathrm{H}$; d, J= 9 $\mathrm{Hz}, \mathrm{AB}$ system), 7.92 ( 2H; d, J= 9 Hz, AB system), 7.96 ( 2H; d,
$\mathrm{J}=9 \mathrm{~Hz}, \mathrm{AB}$ system $), 10.59\left(1 \mathrm{H}, \mathrm{s}\right.$, exchangeable with $\left.\mathrm{D}_{2} \mathrm{O}\right)$; MS $(\mathrm{m} / \mathrm{z}, \%)=273(\mathrm{M}+, 12.74)$. Anal.Calcd. for $\mathrm{C} 15 \mathrm{H} 12 \mathrm{ClNO} 2$ (273.06): C, 65.82; H, 4.42; N, $5.12 \%$. Found C, 65.62; H, 4.32; N, $5.09 \%$.

\section{$N$-(4-Acetylphenyl)-2,4-dichlorobenzamide (3b)}

To a solution of p-aminoacetophenone $(0.01 \mathrm{~mol})$ in dimethyl formamide $(20 \mathrm{~mL})$ an equimolar amount of 2,4-dichloro benzoyl chloride $(0.01 \mathrm{~mol})$ was added. The mixture was stirred at $0^{\circ} \mathrm{C}$ for $3 \mathrm{~h}$, after which crushed ice was added with continuous stirring. A heavy precipitate was obtained, which was filtered, washed with water and after drying it was crystallized out from ethanol. The yield was $63 \%$ and the melting temperature was 170 $171^{\circ} \mathrm{c}$. The IR spectrum, ${ }_{\max }, \mathrm{cm}^{-1}$, was: $3340(\mathrm{NH}), 1694$ (br., $2 \mathrm{C}=\mathrm{O}$ ). The ${ }^{1} \mathrm{H}$ NMR spectrum, (300 MHz, DMSO-d6) $\delta(\mathrm{ppm})$, was: $2.54\left(3 \mathrm{H} ; \mathrm{s}, \mathrm{CH}_{3}\right), 7.51(2 \mathrm{H} ; \mathrm{d}, \mathrm{J}=6 \mathrm{~Hz}, \mathrm{~J}=9 \mathrm{~Hz}, \mathrm{AB}$ system), $7.66(2 \mathrm{H} ; \mathrm{d}, \mathrm{J}=6 \mathrm{~Hz}, \mathrm{~J}=9 \mathrm{~Hz}, \mathrm{AB}$ system $), 7.73-7.80$ $(3 \mathrm{H} ; \mathrm{m}), 10.87\left(1 \mathrm{H}, \mathrm{s}\right.$, exchangeable with $\left.\mathrm{D}_{2} \mathrm{O}\right) ; \mathrm{MS}(\mathrm{m} / \mathrm{z}, \%)=$ 308 (M+, 12.74). Anal.Calcd.for $\mathrm{C}_{15} \mathrm{H}_{11} \mathrm{Cl}_{2} \mathrm{NO}_{2}$ (307.02): C, 58.46; $\mathrm{H}, 3.60 ; \mathrm{N}, 4.55 \%$. Found C, 58.62; H, 3.64; N, $4.61 \%$.

\section{General procedure for synthesis of $N$-(4-3-(pheny)-1-prop-2-en- 1-one phenyl) benzamide derivatives (5a-h)}

A mixture of $\mathrm{N}$-(4-acetylphenyl)-4-chlorobenzamide 3a $(0.01 \mathrm{~mol})$ and an equimolar amount of the appropriate aldehyde was dissolved in $(30 \mathrm{~mL})$ of ethanol. To this mixture, $10 \mathrm{~mL}$ of $10 \% \mathrm{NaOH}\left(10 \mathrm{~g}\right.$ in $\left.100 \mathrm{~mL} \mathrm{H}_{2} \mathrm{O}\right)$ solution, were added drop-wise with continuous stirring for $30 \mathrm{~min}$. Stirring was continued for another 4-5 $\mathrm{h}$ at room temperature, (TLC monitoring). After the reaction was complete, sometimes turbidity appeared, it was diluted with ice-cold distilled water $(40 \mathrm{~mL})$, filtered, washed well with cold water, dried in air and recrystallized from ethanol.

\section{4-Chloro-N-(4-3-(4-chloropheny)-1-prop-2-en-1-one phenyl) benzamide (5a)}

The yield was $40 \%$ and the melting temperature was 203 $204^{\circ} \mathrm{C}$. The IR spectrum, $(\mathrm{KBr}),{ }_{\max }, \mathrm{cm}^{-1}$ was: $3353(\mathrm{NH}), 1650$ (br., $2 \mathrm{C}=\mathrm{O}$ ). The ${ }^{1} \mathrm{H}$ NMR spectrum, (300 MHz, DMSO-d6) $\delta$ (ppm), was:7.51 $(2 \mathrm{H} ; \mathrm{d}), 7.62(1 \mathrm{H} ; \mathrm{d}, \mathrm{J}=8.4 \mathrm{~Hz}, \mathrm{CH}), 7.69-8.03$ $(10 \mathrm{H} ; \mathrm{m}), 8.19(1 \mathrm{H} ; \mathrm{d}, \mathrm{J}=8.4 \mathrm{~Hz}, \mathrm{CH}), 10.62(1 \mathrm{H}, \mathrm{NH}, \mathrm{s}$, exchangeable by $\left.\mathrm{D}_{2} \mathrm{O}\right)$; $\mathrm{MS}(\mathrm{m} / \mathrm{z}, \%)=395(\mathrm{M}+, 34.74)$. Anal.Calcd.for $\mathrm{C}_{22} \mathrm{H}_{15} \mathrm{Cl}_{2} \mathrm{NO}_{2}(395.05)$ : C, 66.68; H, 3.82; N, 3.53 $\%$. Found C, 66.79; H, 3.88; N, $3.67 \%$.

\section{4-Chloro-N-(4-3-(4-floropheny)-1-prop-2-en-1-one phenyl) benzamide $(5 b)$}

The yield was $55 \%$ and the melting temperature was 204 $205^{\circ} \mathrm{C}$. The IR spectrum, $(\mathrm{KBr}), v_{\max }, \mathrm{cm}^{-1}$, was: $3360(\mathrm{NH}), 1656$ (br., $2 \mathrm{C}=\mathrm{O}$ ). The ${ }^{1} \mathrm{H}$ NMR spectrum, (300 MHz, DMSO-d6) $\delta$ (ppm), was: $7.28(2 \mathrm{H} ; \mathrm{d}), 7.61(1 \mathrm{H} ; \mathrm{d}, \mathrm{J}=8.4 \mathrm{~Hz}, \mathrm{CH}), 7.70-8.03$ $(10 \mathrm{H} ; \mathrm{m}), 8.18(1 \mathrm{H} ; \mathrm{d}, \mathrm{J}=8.4 \mathrm{~Hz}, \mathrm{CH}), 10.64(1 \mathrm{H}, \mathrm{NH}, \mathrm{s}$, exchangeable by $\left.\mathrm{D}_{2} \mathrm{O}\right) ; \mathrm{MS}(\mathrm{m} / \mathrm{z}, \%)=379(\mathrm{M}+, 49.12)$. 
4-Chloro-N-(4-3-(2-hydroxypheny)-1-prop-2-en-1-one phenyl) benzamide $(5 \mathrm{c})$

The yield was $43 \%$ and the melting temperature was $198-199^{\circ} \mathrm{C}$. The IR spectrum, (KBr), $v_{\max } \mathrm{cm}^{-1}$, was: $3350(\mathrm{NH}), 1670$ (br., $2 \mathrm{C}=\mathrm{O})$. The ${ }^{1} \mathrm{H}$ NMR spectrum, (300 MHz, DMSO-d6) $\delta(\mathrm{ppm})$, was: $6.23(1 \mathrm{H} ; \mathrm{d}), 6.52(1 \mathrm{H} ; \mathrm{m}), 7.08(1 \mathrm{H}, \mathrm{OH}, \mathrm{s}$, exchangeable by $\left.\mathrm{D}_{2} \mathrm{O}\right), 7.22-7.83(11 \mathrm{H} ; \mathrm{m}, \mathrm{CH}$, Aromatic H), 7,98 $(1 \mathrm{H} ; \mathrm{d}, \mathrm{CH})$, $10.50\left(1 \mathrm{H}, \mathrm{NH}, \mathrm{s}\right.$, exchangeable by $\left.\mathrm{D}_{2} \mathrm{O}\right)$; MS $(\mathrm{m} / \mathrm{z}, \%)=377$ $(\mathrm{M}+, 1.28)$.

\section{4-Chloro-N-(4-3-(4-methylpheny)-1-prop-2-en-1-one phenyl) benzamide $(5 d)$}

The yield was $45 \%$ and the melting temperature was $187-188^{\circ} \mathrm{C}$. The IR spectrum, $(\mathrm{KBr}), v_{\max }, \mathrm{cm}^{-1}$, was: 3365 (NH), 1655 (br., 2C=O). The ${ }^{1} \mathrm{H}$ NMR spectrum, (300 MHz, DMSO-d6) $\delta$ (ppm), was: $2.36\left(3 \mathrm{H}\right.$; s, $\left.\mathrm{CH}_{3}\right), 7.27(2 \mathrm{H} ; \mathrm{d}), 7.60-$ $8.04(11 \mathrm{H} ; \mathrm{m}, \mathrm{CH}$, Aromatic $\mathrm{H}), 8.16(1 \mathrm{H} ; \mathrm{d}, \mathrm{CH}), 10.52$ $\left(1 \mathrm{H}, \mathrm{NH}, \mathrm{s}\right.$, exchangeable by $\left.\mathrm{D}_{2} \mathrm{O}\right) ; \mathrm{MS}(\mathrm{m} / \mathrm{z}, \%)=373(\mathrm{M}+-2$, 2.03).

\section{4-Chloro-N-(4-3-(4-methoxypheny)-1-prop-2-en-1-one phenyl)} benzamide (5e)

The yield was $68 \%$ and the melting temperature was 187 $190^{\circ} \mathrm{C}$. The IR spectrum, $(\mathrm{KBr}), v_{\max } \mathrm{cm}^{-1}$, was: $3350(\mathrm{NH}), 1675$ (br., $2 \mathrm{C}=\mathrm{O}$ ). The ${ }^{1} \mathrm{H}$ NMR spectrum, (300 MHz, DMSO-d6) $\delta$ (ppm), was: $3.86\left(3 \mathrm{H}\right.$; s, $\left.\mathrm{OCH}_{3}\right), 7.11(1 \mathrm{H} ; \mathrm{d}), 7.22-7.88(11 \mathrm{H} ; \mathrm{m}$, $\mathrm{CH}$, Aromatic H), $8.54(1 \mathrm{H} ; \mathrm{d}, \mathrm{CH}), 9.87(1 \mathrm{H}, \mathrm{NH}, \mathrm{s}$, exchangeable by $\left.\mathrm{D}_{2} \mathrm{O}\right)$; $\mathrm{MS}(\mathrm{m} / \mathrm{z}, \%)=391(\mathrm{M}+, 0.66)$. Anal.Calcd. for $\mathrm{C}_{23} \mathrm{H}_{18} \mathrm{ClNO}_{3}$ (391.1) : C, 70.50; H, 4.63; N, 3.57 $\%$. Found C, 70.68; H, 4.69; N, $3.64 \%$.

\section{4-Dichloro- $N$-(4-3-(3,4,5-trimethoxypheny)-1-prop-2-en-1-one phenyl) benzamide (5f)}

The yield was $38 \%$ and the melting temperature was 210 $211^{\circ} \mathrm{C}$. The IR spectrum, $(\mathrm{KBr}), \mathrm{v}_{\max }, \mathrm{cm}^{-1}$, was: $3359(\mathrm{NH}), 1688$ (br., $2 \mathrm{C}=\mathrm{O}$ ). The ${ }^{1} \mathrm{H}$ NMR spectrum, (300 MHz, DMSO-d6) $\delta$ (ppm), was: 3.86 (9H; s, 3OCH3), $6.88(1 \mathrm{H} ; \mathrm{d}), 7.24-7.82(9 \mathrm{H} ; \mathrm{m}$, $\mathrm{CH}$, Aromatic $\mathrm{H}), 8.53(1 \mathrm{H} ; \mathrm{d}, \mathrm{CH}), 9.82(1 \mathrm{H}, \mathrm{NH}, \mathrm{s}$, exchangeable by $\left.\mathrm{D}_{2} \mathrm{O}\right)$; $\mathrm{MS}(\mathrm{m} / \mathrm{z}, \%)=452(\mathrm{M}+, 1.28)$. Anal. Calcd.for $\mathrm{C}_{25} \mathrm{H}_{22} \mathrm{ClNO}_{5}$ ( 451.12) : C, 66.45; H, 4.91; N, $3.10 \%$. Found C, 66.58; H, 4.88; N, $3.19 \%$.

\section{4-Dichloro-N-(4-3-(3-hydroxy-4-methoxypheny)-1-prop-2-en-1- one phenyl) benzamide (5g)}

The yield was $60 \%$ and the melting temperature was $219-220^{\circ} \mathrm{C}$. The IR spectrum, $(\mathrm{KBr}), v_{\max }, \mathrm{cm}^{-1}$, was: 3351 (NH), 1655 (br., 2C=O). The ${ }^{1} \mathrm{H}$ NMR spectrum, $\quad(300$ MHz, DMSO-d6) $\delta$ (ppm), was: $3.83(3 \mathrm{H}$; s, OCH3), $5.63(1 \mathrm{H}$, $\mathrm{OH}, \mathrm{s}$, exchangeable by $\left.\mathrm{D}_{2} \mathrm{O}\right), 7.61-7.64(4 \mathrm{H} ; \mathrm{m}, \mathrm{CH}$, Aromatic H), 7.90-8.02 (9H; m, CH, Aromatic H), 10.60 $\left(1 \mathrm{H}, \mathrm{NH}, \mathrm{s}\right.$, exchangeable by $\left.\mathrm{D}_{2} \mathrm{O}\right) ; \mathrm{MS}(\mathrm{m} / \mathrm{z}, \%)=407$ (M+, 1.28). Anal. Calcd. for $\mathrm{C}_{23} \mathrm{H}_{18} \mathrm{ClNO}_{4}$ ( 407.09): C, $67.73 ; \mathrm{H}, 4.45 ; \mathrm{N}, 3.43 \%$. Found C, 67.90; H, 4.48; N, $3.40 \%$.

\section{4-Dichloro- $\mathrm{N}$-(4-3-(furan-2-yl)-1-prop-2-en-1-one phenyl) benzamide $(5 h)$}

The yield was $35 \%$ and the melting temperature was 221 $222^{\circ} \mathrm{C}$. The IR spectrum, $(\mathrm{KBr}), v_{\max }, \mathrm{cm}^{-1}$, was: $3335(\mathrm{NH}), 1653$ (br., $2 \mathrm{C}=\mathrm{O}$ ). The ${ }^{1} \mathrm{H}$ NMR spectrum, (300 MHz, DMSO-d6) $\delta$ (ppm), was: 6.69-6.71 $(1 \mathrm{H} ; \mathrm{m}), 7.09(1 \mathrm{H} ; \mathrm{d}, \mathrm{CH}), 7.57-7.65(3 \mathrm{H}$; $\mathrm{m}, \mathrm{CH}$, Aromatic H), 7.83-8.10 (7H; m , CH,Aromatic $\mathrm{H}), 8.13$ $(\mathrm{H}$; d, Aromatic $\mathrm{H}), 10.64\left(1 \mathrm{H}, \mathrm{NH}\right.$, s, exchangeable by $\left.\mathrm{D}_{2} \mathrm{O}\right)$; $\mathrm{MS}$ $(\mathrm{m} / \mathrm{z}, \%)=351(\mathrm{M}+, 40.27)$. Anal. Calcd.for $\mathrm{C}_{20} \mathrm{H}_{14} \mathrm{ClNO}_{3}$ (351.07) : C, 68.28; H, 4.01; N, $3.98 \%$. Found C, 68.41; H, 4.03; $\mathrm{N}, 4.06 \%$.

General procedure for synthesis of N-(4-3-(pheny)-1-prop-2-en1-one phenyl) benzamide derivatives (5i-p)

A mixture of $\mathrm{N}$-(4-acetylphenyl)-2,4-dichlorobenzamide 3b $(0.01 \mathrm{~mol})$ and $(0.01 \mathrm{~mol})$ of the appropriate aldehyde was dissolved in $(30 \mathrm{~mL})$ of ethanol in presence of $10 \%$ sodium hydroxide $\left(10 \mathrm{~g}\right.$ in $\left.100 \mathrm{~mL} \mathrm{H}_{2} \mathrm{O}\right)$. Then stirred at room temperature for $4 \mathrm{~h}$. At the end of the reaction, the mixture was diluted with iced-water, the solid obtained was filtered, washed with water, dried and recrystallized from ethanol.

\section{2,4-Dichloro-N-(4-3-(4-chloropheny)-1-prop-2-en-1-one phenyl) benzamide (5i)}

The yield was $45 \%$ and the melting temperature was 128 $130^{\circ} \mathrm{C}$. The IR spectrum, $(\mathrm{KBr}), v_{\max }, \mathrm{cm}^{-1}$, was: $3290(\mathrm{NH}), 1667$ (br., $2 \mathrm{C}=\mathrm{O}$ ). The ${ }^{1} \mathrm{H}$ NMR spectrum, (300 MHz, DMSO-d6) $\delta$ (ppm), was: 7.19-7.84 (12H; m, CH, Aromatic H), $7.99(\mathrm{~d} ; \mathrm{H}, \mathrm{CH})$, $8.40\left(1 \mathrm{H}, \mathrm{NH}, \mathrm{s}\right.$, exchangeable by $\left.\mathrm{D}_{2} \mathrm{O}\right) ; \mathrm{MS}(\mathrm{m} / \mathrm{z}, \%)=429(\mathrm{M}+$, 1.72). Anal.Calcd.for $\mathrm{C}_{22} \mathrm{H}_{14} \mathrm{Cl}_{3} \mathrm{NO}_{2}(429.01)$ : $\mathrm{C}, 61.35 ; \mathrm{H}, 3.28$; $\mathrm{N}, 3.25 \%$. Found C, 61.43; H, 3.26; N, 3.32\%.

\section{2,4-Dichloro- $N$-(4-3-(4-floropheny)-1-prop-2-en-1-one phenyl) benzamide (5j)}

The yield was $55 \%$ and the melting temperature was 200 $202^{0} \mathrm{C}$. The IR spectrum, $(\mathrm{KBr}), v_{\max }, \mathrm{cm}^{-1}$, was: $3411(\mathrm{NH}), 1660$ (br., $2 \mathrm{C}=\mathrm{O}$ ). The ${ }^{1} \mathrm{H}$ NMR spectrum, (300 MHz, DMSO-d6) $\delta$ (ppm), was: 7.20-7.44 (5H; m, $\mathrm{CH}$, Aromatic $\mathrm{H}), 7.46-7.88(8 \mathrm{H}$; $\mathrm{m}, \mathrm{CH}$, Aromatic $\mathrm{H}), 8.40\left(1 \mathrm{H}, \mathrm{NH}\right.$, s, exchangeable by $\left.\mathrm{D}_{2} \mathrm{O}\right)$; $\mathrm{MS}$ $(\mathrm{m} / \mathrm{z}, \%)=413\left(\mathrm{M}^{-1}, 0.30\right)$. Anal.Calcd.for $\mathrm{C}_{22} \mathrm{H}_{14} \mathrm{Cl}_{2} \mathrm{FNO}_{2}($ 413.04) : C, 63.79; H, 3.41; N, 3.38\%. Found C, 63.91; H, 3.46; N, $3.42 \%$.

\section{2,4-Dichloro-N-(4-3-(2-hydroxypheny)-1-prop-2-en-1-one phenyl) benzamide ( $5 k$ )}

The yield was $57 \%$ and the melting temperature was $>$ $300^{\circ} \mathrm{C}$. The IR spectrum, $(\mathrm{KBr}), v_{\max }, \mathrm{cm}^{-1}$, was: $3412(\mathrm{NH}), 1671$ (br., 2=CO). The ${ }^{1} \mathrm{H}$ NMR spectrum, (300 MHz, DMSO-d6) $\delta$ $(\mathrm{ppm})$, was: $6.21(1 \mathrm{H} ; \mathrm{d}), 6.49(1 \mathrm{H} ; \mathrm{m}), 7.08(1 \mathrm{H}, \mathrm{OH}, \mathrm{s}$, exchangeable by $\left.\mathrm{D}_{2} \mathrm{O}\right), 7.22-7.40(5 \mathrm{H} ; \mathrm{m}, \mathrm{CH}$, Aromatic $\mathrm{H}), 7.52$ $(2 \mathrm{H} ; \mathrm{d}, \mathrm{J}=8.4 \mathrm{~Hz}, \mathrm{AB}$ system $), 7.64(2 \mathrm{H} ; \mathrm{d}, \mathrm{J}=8.4 \mathrm{~Hz}, \mathrm{AB}$ system), $7.74(1 \mathrm{H} ; \mathrm{s}), 7.98(1 \mathrm{H} ; \mathrm{d}, \mathrm{CH}), 8.50(\mathrm{~s}, 1 \mathrm{H}, \mathrm{NH}$, exchangeable by $\left.\mathrm{D}_{2} \mathrm{O}\right)$; MS $(\mathrm{m} / \mathrm{z}, \%)=411(\mathrm{M}+, 0.52)$. 
Anal.Calcd.for $\mathrm{C}_{22} \mathrm{H}_{15} \mathrm{Cl}_{2} \mathrm{NO}_{3}$ (411.04) : C, 64.09; $\mathrm{H}, \mathrm{H}, 3.67 ; \mathrm{N}$, $3.40 \%$. Found C, 64.24; H, 3.72; N, 3.49\%.

\section{2,4-Dichloro-N-(4-3-(4-methylpheny)-1-prop-2-en-1-one phenyl) benzamide (5l)}

The yield was $60 \%$ and the melting temperature was 148 $150^{\circ} \mathrm{C}$. The IR spectrum, $(\mathrm{KBr}), v_{\max }, \mathrm{cm}^{-1}, 3299(\mathrm{NH}), 1664$ (br., $2 \mathrm{C}=\mathrm{O}$ ). The ${ }^{1} \mathrm{H}$ NMR spectrum, (300 MHz, DMSO-d6) $\delta(\mathrm{ppm})$, was: $2.36\left(3 \mathrm{H} ; \mathrm{s}, \mathrm{CH}_{3}\right), 7.21-7.91(13 \mathrm{H} ; \mathrm{m}, 2 \mathrm{CH}$, Aromatic $\mathrm{H})$, $8.17\left(\mathrm{~s}, 1 \mathrm{H}, \mathrm{NH}\right.$, exchangeable by $\left.\mathrm{D}_{2} \mathrm{O}\right) ; \mathrm{MS} \quad(\mathrm{m} / \mathrm{z}, \%)=409$ $\left(\mathrm{M}+\right.$, 42.87). Anal.Calcd. for $\mathrm{C}_{23} \mathrm{H}_{17} \mathrm{Cl}_{2} \mathrm{NO}_{2}$ (409.06) : C, 67.33; $\mathrm{H}$, $4.18 ; \mathrm{N}, 3.41 \%$. Found C, 67.23; H, 4.11; N, 3.21\%.

\section{2,4-Dichloro-N-(4-3-(4-methoxypheny)-1-prop-2-en-1-one phenyl) benzamide (5m)}

The yield was $65 \%$ and the melting temperature was 230 $233^{\circ} \mathrm{C}$. The IR spectrum, $(\mathrm{KBr}), v_{\max }, \mathrm{cm}^{-1}$, was: $3412(\mathrm{NH}), 1660$ (br., 2C=O). The ${ }^{1} \mathrm{H}$ NMR spectrum, (300 MHz, DMSO-d6) $\delta$ (ppm), was: $3.83(3 \mathrm{H} ; \mathrm{s}, \mathrm{OCH} 3), 7.01(2 \mathrm{H} ; \mathrm{d}, \mathrm{J}=9 \mathrm{~Hz}, \mathrm{AB}$ system), 7.22-7.34 (3H; m), $7.55(1 \mathrm{H} ; \mathrm{d}, \mathrm{J}=8.4 \mathrm{~Hz}, \mathrm{CH}), 7.66$ $(2 \mathrm{H} ; \mathrm{d}, \mathrm{J}=9 \mathrm{~Hz}, \mathrm{AB}$ system), 7.73-7.90 (m; 4H), $8.16(1 \mathrm{H} ; \mathrm{d}, \mathrm{J}=$ $8.4 \mathrm{~Hz}, \mathrm{CH}), 11.01\left(\mathrm{~s}, 1 \mathrm{H}, \mathrm{NH}\right.$, exchangeable by $\left.\mathrm{D}_{2} \mathrm{O}\right) ; \mathrm{MS}(\mathrm{m} / \mathrm{z}$, $\%)=425(\mathrm{M}+, 21.88)$. Anal.Calcd.for $\mathrm{C}_{23} \mathrm{H}_{17} \mathrm{Cl}_{2} \mathrm{NO}_{3}(425.06):$ C, 64.80; H, 4.02; N, $3.29 \%$. Found C, 64.95; H, 4.08; N, $3.36 \%$.

\section{2,4-Dichloro-N-(4-3-(3,4,5-trimethoxypheny)-1-prop-2-en-1-one phenyl) benzamide (5n)}

The yield was 55\% and the melting temperature was 148 $150^{\circ} \mathrm{C}$. The IR spectrum, $(\mathrm{KBr}), v_{\max }, \mathrm{cm}^{-1}$, was: $3393(\mathrm{NH}), 1664$ (br., 2C=O). The ${ }^{1} \mathrm{H}$ NMR spectrum, (300 MHz, DMSO-d6) $\delta$ (ppm), was: $3.86\left(9 \mathrm{H} ; \mathrm{s}, 3 \mathrm{OCH}_{3}\right), 6.26-7.89(11 \mathrm{H} ; \mathrm{m}, 2 \mathrm{CH}$, Aromatic $\mathrm{H}), 10.01\left(\mathrm{~s}, 1 \mathrm{H}, \mathrm{NH}\right.$, exchangeable by $\left.\mathrm{D}_{2} \mathrm{O}\right) ; 7.37 \mathrm{MS}$ $(\mathrm{m} / \mathrm{z}, \%)=486(\mathrm{M}+1,45.70)$. Anal.Calcd. for $\mathrm{C}_{25} \mathrm{H}_{21} \mathrm{Cl}_{2} \mathrm{NO}_{5}$ (485.08) : C, 61.74; H, 4.35; N, 2.88\%. Found C, 61.58; H, 4.15; $\mathrm{N}, 2.67 \%$.

\section{2,4-Dichloro-N-(4-3-(3-hydroxy-4-methoxypheny)-1-prop-2-en- 1-one phenyl) benzamide (5o)}

The yield $60 \%$ and the melting temperature was 245 $248^{0} \mathrm{C}$. The IR spectrum, $(\mathrm{KBr}), v_{\max }, \mathrm{cm}^{-1}$, was: $3409(\mathrm{NH}), 1669$ (br., 2C=O). The ${ }^{1} \mathrm{H}$ NMR spectrum, (300 MHz, DMSO-d6) $\delta$ (ppm), was: $2.54\left(\mathrm{~s}, 1 \mathrm{H}, \mathrm{OH}\right.$, exchangeable by $\left.\mathrm{D}_{2} \mathrm{O}\right), 3.97(3 \mathrm{H} ; \mathrm{s}$, $\left.\mathrm{OCH}_{3}\right), 6.58(1 \mathrm{H}, \mathrm{d}), 7.15(1 \mathrm{H}, \mathrm{s}), 7.21(1 \mathrm{H}, \mathrm{d}), 7.24-7.36(3 \mathrm{H} ; \mathrm{m})$, $7.54(2 \mathrm{H} ; \mathrm{d}, \mathrm{J}=7.2 \mathrm{~Hz}, \mathrm{AB}$ system $), 7.65(2 \mathrm{H} ; \mathrm{d}, \mathrm{J}=7.2 \mathrm{~Hz}, \mathrm{AB}$ system), $7.65(1 \mathrm{H} ; \mathrm{d}, \mathrm{J}=9 \mathrm{~Hz}, \mathrm{CH}), 7.99(1 \mathrm{H} ; \mathrm{d}, \mathrm{J}=9 \mathrm{~Hz}, \mathrm{CH})$, $10.98\left(\mathrm{~s}, 1 \mathrm{H}, \mathrm{NH}\right.$, exchangeable by $\left.\mathrm{D}_{2} \mathrm{O}\right) ; \mathrm{MS} \quad(\mathrm{m} / \mathrm{z}, \%)=443$ $\left(\mathrm{M}^{+}+2\right.$, 12.77). Anal.Calcd. for $\mathrm{C}_{23} \mathrm{H}_{17} \mathrm{Cl}_{2} \mathrm{NO}_{4}$ (441.01) : $\mathrm{C}, 62.46$; $\mathrm{H}, 3.87 ; \mathrm{N}, 3.17 \%$. Found C, 62.23; H, 3.65; N, 3.09\%.

\section{2,4-Dichloro-N-(4-3-(furan-2-yl)-1-prop-2-en-1-one phenyl) benzamide (5p)}

The yield was 50\% and the melting temperature was 242$245^{\circ} \mathrm{C}$. The IR spectrum, $(\mathrm{KBr}), v_{\max }, \mathrm{cm}^{-1}$, was: $3413(\mathrm{NH}), 1660$ (br., $2 \mathrm{C}=\mathrm{O}$ ). The ${ }^{1} \mathrm{H}$ NMR spectrum, (300 MHz, DMSO-d6) $\delta$ (ppm), was: $6.69(1 \mathrm{H} ; \mathrm{m}), 7.20-7.88(9 \mathrm{H} ; \mathrm{m}, \mathrm{CH}$, Aromatic $\mathrm{H})$, $7.90(1 \mathrm{H} ; \mathrm{d}, \mathrm{CH}), 8.09(1 \mathrm{H} ; \mathrm{s}), 10.01(\mathrm{~s}, 1 \mathrm{H}, \mathrm{NH}$, exchangeable by $\left.\mathrm{D}_{2} \mathrm{O}\right) ; \mathrm{MS}(\mathrm{m} / \mathrm{z}, \%)=386\left(\mathrm{M}^{+}+1,32.35\right)$. Anal.Calcd. for $\mathrm{C}_{20} \mathrm{H}_{13} \mathrm{Cl}_{2} \mathrm{NO}_{3}$ (385.03): C, 62.19; H, 3.39; N, $3.63 \%$. Found C, $62.49 ; \mathrm{H}, 3.59 ; \mathrm{N}, 3.83 \%$.

\section{Biology}

Cytotoxicity assay against PC-3 Prostate Adenocarcinoma Cell Line: Cell lines and treatment: The inhibitory effect of the new synthesized compounds on human prostate cancer PC-3 was performed at the pharmacology department, Ain Shams University, Cairo, Egypt.

\section{Cell culture}

The human prostate cancer cells, PC3 were grown in RPMI-1640 medium, supplemented with $10 \%$ heat inactivated FBS, 100 units $/ \mathrm{ml}$ penicillin and $100 \mathrm{mg} / \mathrm{mL}$ streptomycin and was maintained at $37^{\circ}$ in a humidified atmosphere containing $5 \%$ $\mathrm{CO} 2$. The cells were maintained as a "monolayer culture" by serial sub-culturing.

\section{Sulforhodamine B (SRB) cytotoxicity assay}

Cytotoxicity was determined using the SRB method (Skehan et al., 1990). Exponentially growing cells were then collected using $0.25 \%$ Trypsin-EDTA and seeded in a 96-well plates at 1000-2000 cells/well in RPMI-1640 supplemented medium. After $24 \mathrm{~h}$, the cells were incubated for $72 \mathrm{~h}$ with different concentrations of the tested compounds. Following $72 \mathrm{~h}$ treatment, the cells were fixed with $10 \%$ trichloroacetic acid for 1 $\mathrm{h}$ at $4{ }^{\circ} \mathrm{C}$. The wells were stained at $\mathrm{r}$. t. for $10 \mathrm{~min}$ with $0.4 \%$ SRB dissolved in $1 \%$ acetic acid.

The plates were then air dried for $24 \mathrm{~h}$ and the dye was solubilized with Tris- $\mathrm{HCl}$ for $5 \mathrm{~min}$ on a shaker at $1600 \mathrm{rpm}$. Optical density (OD) of each well was measured spectrophotometrically at $564 \mathrm{~nm}$ with an ELISA microplate reader (ChroMate-4300, FL, USA). IC50 values were calculated according to the equation for Boltzman sigmoidal concentrationresponse curve using the nonlinear regression fitting models (Graph Pad, Prism Version 5).

\section{Cathepsin B Assay}

1 The cell culture PC3 human prostate cancer cells were grown in RPMI-1640 medium supplemented with $10 \%$ heat inactivated FBS, 100 units $/ \mathrm{mL}$ of penicillin and 100 $\mathrm{mg} / \mathrm{mL}$ of streptomycin and maintained at $37^{\circ}$ in a humidified atmosphere containing $5 \% \quad \mathrm{CO} 2$. Exponentially growing cells were seeded in 6-well plates at $(4 \times 105)$ cells/well in an RPMI-1640 supplemented medium. After $24 \mathrm{~h}$, cells were incubated for $24 \mathrm{~h}$ with the tested compounds at their median inhibitory concentrations (IC50). Then, cells were washed with $1 \mathrm{x}$ PBS, scraped, homogenized in ice-cold PBS using Dounce homogenizer and stored overnight at $-20^{\circ} \mathrm{C}$. After two freeze-thaw cycles, the cells were centrifuged 
at $5000 \times \mathrm{g}$ for 5 minutes. Supernatants were collected and immediately assayed.

2 ELISA assay cathepsin-B (CTS-B) was quantified in the collected samples using enzyme-linked immunosorbent assay kit (Wuhan ElAab Science Co., Ltd, Wuhan, China) according to the manufacturer instructions. The assay was based on the sandwich technique in which specific antibodies to CTS-B were pre-coated onto 96well plate. The specific detection antibodies were biotinylated. The test samples and biotinylated detection antibodies were added sequentially followed by washing. Avidin-Peroxidase Complex was added and unbound conjugates were then washed. A substrate solution was added to the wells to determine the bound enzyme activity. The color development was stopped by sulphuric acid, and the absorbance was read at $450 \mathrm{~nm}$ using an ELISA microplate reader (ChroMate-4300, FL, USA). The intensity of the color was directly proportional to the concentration of CTS-B in the sample.

\section{RESULTS AND DISCUSSION}

\section{Chemistry}

Synthesis of the novel target compounds were accomplished following the procedures depicted in schemes 1 and 2. The intermediates $\mathbf{3 a} \mathbf{a}, \mathbf{b}$ were prepared by benzoylation of the free amino of the acetophenone using either 4-chloro or 2,4dichloro benzoyl chloride derivatives. The target compounds 5a-p were obtained by the, Claisen-Schmidt aldol condensation in basic conditions, of the two acetophenone derivatives $\mathbf{3 a , b}$ with the appropriate aldehyde (Sunduru et al., 2006). The spectral data were consistent with their expected structures.<smiles>[R7]c1ccc(C(=O)Nc2ccc(C(C)=O)cc2)cc1</smiles>

$1 a, b$

2

$3 a, b$

1a,3a: $R_{1}=4-C l$,

1b,3b: $R_{1}=2,4-d i ~ C l$

Reagents and conditions: i, 2,4-dichlorobenzoyl chloride or 4-chlorobenzoyl chloride/DMF $/ 0^{\circ} \mathrm{C}$

Scheme1: Synthesis of the key synthons N-(4-acetylphenyl) benzamide 3a,b.<smiles>[R7]c1ccc(C(=O)Nc2ccc(C(C)=O)cc2)cc1</smiles>

$3 a, b$

3a $\mathrm{R}_{1}=4-\mathrm{Cl}$ 3b $R_{1}=2,4$-di Cl
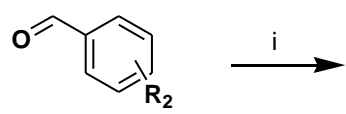

4a-h

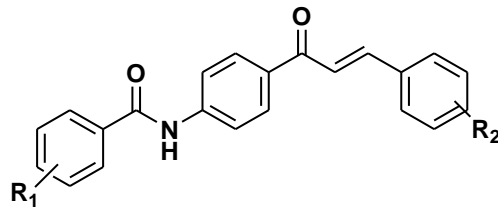

5a-p

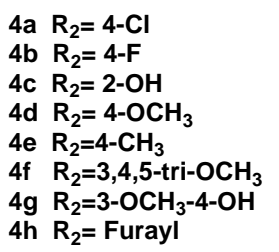

5a $R_{1}=R_{2}=4-C l$

5b $R_{1}=4-C l, R_{2}=4-F$

5c $R_{1}=4-\mathrm{Cl}, \mathrm{R}_{2}=2-\mathrm{OH}$

5d $\mathrm{R}_{1}=4-\mathrm{Cl}, \mathrm{R}_{2}=4-\mathrm{OCH}_{3}$

5e $R_{1}=4-\mathrm{Cl}, \mathrm{R}_{2}=4-\mathrm{CH} 3$

5f $\mathbf{R}_{\mathbf{1}}=4-\mathrm{Cl}, \mathrm{R}_{\mathbf{2}}=3,4,5-\mathrm{tri}-\mathrm{OCH}_{3}$

$5 \mathrm{~g} \mathrm{R}_{1}=4-\mathrm{Cl}, \mathrm{R}_{2}=3-\mathrm{OCH}_{3}-4-\mathrm{OH}$

5 h $R_{1}=4-C l, R_{2}=$ Furyl

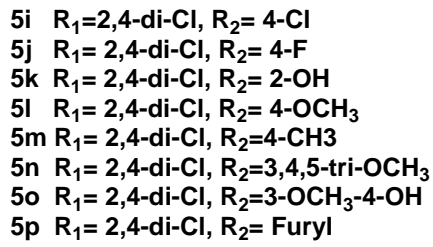

5i $R_{1}=2,4-d i-C l, R_{2}=4-C l$

5j $R_{1}=2,4-d i-C l, R_{2}=4-F$

$5 k \quad R_{1}=2,4-d i-C l, R_{2}=2-O H$

5I $R_{1}=2,4$-di-Cl, $R_{2}=4-O_{3}$ $5 \mathrm{~m} \mathrm{R} \mathrm{R}_{1}=2,4-\mathrm{di}-\mathrm{Cl}, \mathrm{R}_{2}=4-\mathrm{CH} 3$

5n $R_{1}=2,4$-di-Cl, $R_{2}=3,4,5$-tri- $\mathrm{OCH}_{3}$

5o $R_{1}=2,4-d i-C l, R_{2}=3-\mathrm{OCH}_{3}-4-\mathrm{OH}$

5p $R_{1}=2,4-d i-C l, R_{2}=$ Furyl 


\section{Anticancer Activities}

In the present study sixteen novel chalcone derivatives were synthesized and evaluated for their cytotoxic effects against the prostate cancer cell line (PC3), using in-vitro sulforhodamine$\mathrm{B}$ (SRB) method. The screening was carried out by evaluation of the growth inhibition percent using five different doses from which the IC50 values were calculated. The reference drug used was Doxorubicin, IC50 was $0.93 \mu \mathrm{M}$. The results are presented in Table 1. Activities of the novel chalcones in this study was influenced by the different substituents as well as their positions. The results showed that the best activity was demonstrated by the furyl derivative $\mathbf{5 p}$ (IC50 $=5.597 \mu \mathrm{M})$, additionally the (4flourophenyl) acryloylderivative $\mathbf{5 b}$ and the (4-tolyl) acryloyl derivative $\mathbf{5 m}$, have also demonstrated good cytotoxic activity (IC50 = 9.06 and $8.84 \mu \mathrm{M}$, respectively). Moreover, moderate activities were observed for compounds $\mathbf{5 e}, \mathbf{5 g}, \mathbf{5 l}$ and $\mathbf{5 0}(18.18$, 19.82, 12.83 and $17.32 \mu \mathrm{M}$, respectively). The chalcone derivatives of 2-hydroxy-3-methoxyphenyl, 5g and 5o, showed close cytotoxic effects suggesting that this moiety greatly controlled the activity. Furthermore, chalcone derivatives carrying (4-chlorophenyl) acryloyl5a, 5i, (3,4,5-trimethoxyphenyl) acryloyl $\mathbf{5 f}, \mathbf{5 n}$ or (2-hydroxy) acryloyl $\mathbf{5 c}, \mathbf{5 k}$, were devoid of any activity. It could be also observed that the activity of compound $\mathbf{5 p}$ with 2,4-dichlorobenzamide was 16-folds more active than its counterpart 4-chloro derivative $\mathbf{5 h}$ (IC50 $=5.597,84.93 \mu \mathrm{M}$, respectively).

Table 1: Cytotoxicity of the studied compounds towards prostate cancer cell line, $\mathrm{PC} 3$.

\begin{tabular}{cccccccc}
$\begin{array}{c}\text { Cpd. } \\
\text { No. }\end{array}$ & $\begin{array}{c}\text { IC50 } \\
\text { PC-3 } \\
(\boldsymbol{\mu M})\end{array}$ & $\begin{array}{c}\text { Cpd. } \\
\text { No. }\end{array}$ & $\begin{array}{c}\text { IC50 } \\
\text { PC-3 } \\
(\boldsymbol{\mu M})\end{array}$ & $\begin{array}{c}\text { Cpd. } \\
\text { No. }\end{array}$ & $\begin{array}{c}\text { IC50 } \\
\text { PC-3 } \\
(\boldsymbol{\mu M})\end{array}$ & $\begin{array}{c}\text { Cpd. } \\
\text { No. }\end{array}$ & $\begin{array}{c}\text { IC50 } \\
\text { PC-3 } \\
(\boldsymbol{\mu M})\end{array}$ \\
\hline $\mathbf{5 a}$ & 151.3 & $\mathbf{5 e}$ & 18.18 & $\mathbf{5 i}$ & 982.6 & $\mathbf{5 m}$ & 9.06 \\
$\mathbf{5 b}$ & 8.84 & $\mathbf{5 f}$ & 332.1 & $\mathbf{5 j}$ & 152.0 & $\mathbf{5 n}$ & 75.67 \\
$\mathbf{5 c}$ & 109.1 & $\mathbf{5 g}$ & 19.82 & $\mathbf{5 k}$ & 100.6 & $\mathbf{5 o}$ & 17.32 \\
$\mathbf{5 d}$ & 302.8 & $\mathbf{5 h}$ & 84.93 & $\mathbf{5 l}$ & 12.83 & $\mathbf{5 p}$ & 5.59 \\
Doxorubicin & 0.925 & & & & & & \\
\hline
\end{tabular}

\section{Cathepsin B inhibition assay}

In the present work, the compounds that showed activity on prostate PC3 cell line; 5b, 5e, 5g, 5l, 5m, 5o and 5p, were further evaluated for their ability to inhibit cathepsin B, using enzyme-linked immunosorbent assay (ELISA) kit (Wuhan ElAab Science Co., Ltd, Wuhan, China), at a concentration equivalent to IC50 for each compound, as presented in Table 2. The flouro derivative $\mathbf{5 b}$ demonstrated the best result, showing 56.6 percent reduction in concentration of the enzyme at dose level $8.849 \mu \mathrm{M}$. Additionally, compounds $\mathbf{5 m}$ and $\mathbf{5 p}$ showed inhibitory effect, $53.2 \%$ and $50.4 \%$, respectively.

Table 2: Growth inhibition percent of the selected compounds against cathepsin B enzyme.

\begin{tabular}{cccc}
\hline Cpd. No. & inhibition (\%) & Cpd.No. & inhibition (\%) \\
\hline $5 \mathrm{~b}$ & 56.6 & $5 \mathrm{~m}$ & 53.2 \\
$5 \mathrm{e}$ & 39.6 & $5 \mathrm{o}$ & 39.5 \\
$5 \mathrm{~g}$ & 33.3 & $5 \mathrm{p}$ & 50.4 \\
$5 \mathrm{l}$ & 45 & & \\
\hline
\end{tabular}

\section{In silico Studies}

3D-QSAR pharmacophore modeling

The 3D QSAR Pharmacophore Generation protocol (Catalyst HypoGen algorithm) was applied to deduce 3D-QSAR pharmacophore models. The HypoGen algorithm identifies the common features in the active compounds and concomitantly excludes those that are common in the inactive ones, within a conformationally allowable region.

It can also estimate the activity of the new compounds by applying regression parameters. Thus, a training set of the 16 synthesized compounds were used in this study to construct a pharmacophore model using hydrogen bond acceptor, hydrogen bond donor, hydrophobic, ring aromatic and positive ionizable features.

Ten top-scored hypotheses were produced based on the activities of the training set. The hypotheses contained four features: HBD, HBA, HYP and RA. Hypo10 consisted of HBD, HBA, HYP and RA, as shown in Fig. 1, the constraint distances and angles between the features are described in Table 3, it also established the highest cost difference (108.08), lower errors (86.70), best correlation coefficient (0.72), maximum fit value (11.17) and lowest root mean square RMS of (2.45). This generated hypothesis, was developed with a total cost value (108.08), a null cost value (139.18) and a fixed cost value (51.60). The fixed total cost depends on the summation of the cost components which are: weight cost, error cost and configuration cost. For the cost analysis, two important values were applied: one was the difference between the fixed and null costs and another one was the difference between the null and total cost (cost difference).

The difference between these two costs should be greater than or equal to 70 bits to show a $98 \%$ statistical significance of the model. While, the cost difference should be greater than 60 bits in order to represent the real correlation data.

When comparing Hypo10 with the other ones, it was observed that it had higher cost difference and correlation value with low RMS and error values. Therefore, Hypo10 was chosen as the best hypothesis and was used for further analysis, the chemical features of Hypo10 and its geometric parameters are shown in Fig. 1. Moreover, the most active compound $\mathbf{5 b}$ was aligned in Hypo10 and was shown in Fig. 2. The prediction accuracy of Hypo10, was also verified by its ability to estimate the activities of compounds within their activity ranges, as presented in Table 4.

Table 3: Constraint distances and angles between features of the generated top pharmacophore model.

\begin{tabular}{|c|c|}
\hline Constraint distances $\left(\mathbf{A}^{\mathbf{0}}\right)$ & Constraint angles $\left({ }^{\circ}\right)$ \\
\hline (HYP -HBD) : 8.61 & HBA,HBD, HYP: 48.49 \\
\hline (HYP -HBA) : 6.48 & HBA, HYP,RA: 59.73 \\
\hline$($ HYP -RA) : 11.95 & HBA, HBD,RA: 169.76 \\
\hline$(\mathrm{HBD}-\mathrm{HBA}): 6.42$ & \\
\hline$(\mathrm{HBD}-\mathrm{RA}): 3.94$ & \\
\hline$(\mathrm{HBA}-\mathrm{RA}): 10.33$ & \\
\hline
\end{tabular}




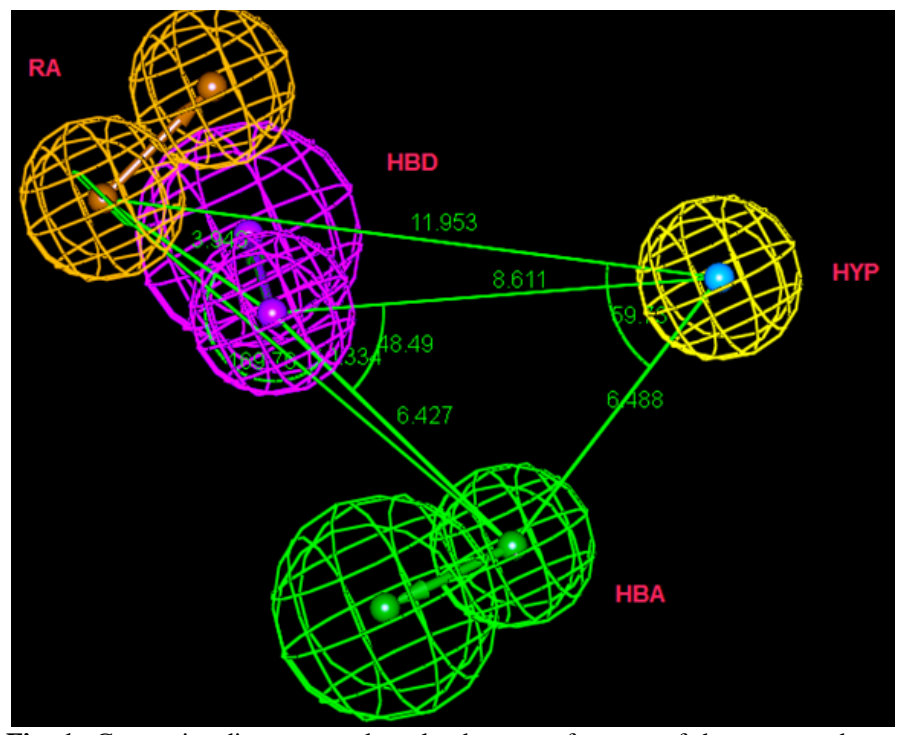

Fig. 1: Constraint distances and angles between features of the generated top pharmacophore model with the features considered hydrogen bond acceptor (HBA) colored in green, hydrogen bond donor (HBD) colored in magenta and aromatic ring (RA) colored in orange, hydrophobic (HYP) colored in cyan.

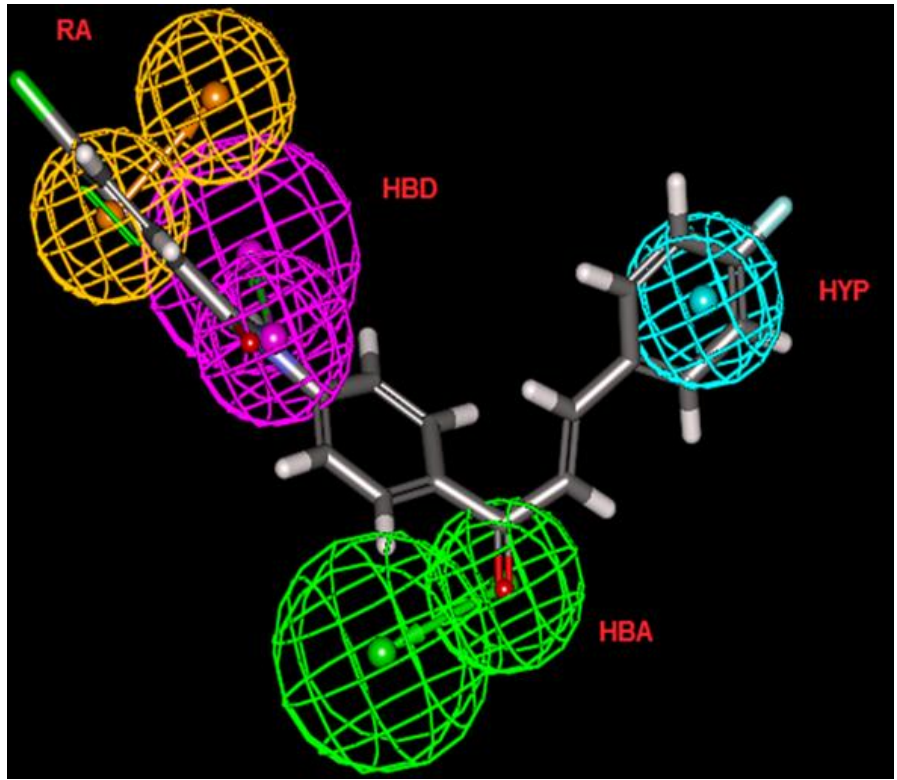

Fig. 2: The best generated pharmacophore hypothesis with the features considered hydrogen bond acceptor (HBA) colored in green, hydrogen bond donor (HBD) colored in magenta and aromatic ring (RA) colored in orange, hydrophobic (HYP) colored in cyan and the synthesized structure $\mathbf{5 b}$ fitted in the pharmacophore with fit value 10.98 .

Fischer validation confidence level selected was 98\%, and the significance of the best generated pharmacophore was 94\%. The generated pharmacophore models were evaluation based on the correlation coefficient, 0.72 , which is considered as a good predictive power. The model was validated by the activity prediction of the synthesized compounds in the training set, in addition to cost analysis and Fischer validation, Table 4.
Table 4: Fit values and estimated activities for the synthesized compounds mapped with the generated 3D-pharmacophore model.

\begin{tabular}{cccc}
\hline Cpd. No. & $\begin{array}{c}\text { Predicted } \\
\text { activity } \\
\left(\mathbf{I C}_{\mathbf{5 0}} \boldsymbol{\mu M}\right)\end{array}$ & $\begin{array}{c}\text { Experimental } \\
\text { activity } \\
\left(\mathbf{I C}_{\mathbf{5 0}} \boldsymbol{\mu} \mathbf{M}\right)\end{array}$ & $\begin{array}{c}\text { Fit } \\
\text { values }\end{array}$ \\
\hline 5a & 95.65 & 151.3 & 10.61 \\
$5 \mathrm{~b}$ & 41.00 & 8.84 & 1098 \\
$5 \mathrm{c}$ & 29.02 & 109.1 & 11.13 \\
$5 \mathrm{~d}$ & 56.18 & 302.8 & 10.84 \\
$5 \mathrm{e}$ & 73.59 & 18.18 & 10.72 \\
$5 \mathrm{f}$ & 67.55 & 332.1 & 10.76 \\
$5 \mathrm{~g}$ & 19.82 & 195.35 & 7.30 \\
$5 \mathrm{~h}$ & 113.33 & 84.93 & 10.54 \\
$5 \mathrm{i}$ & 308.49 & 982.6 & 10.10 \\
$5 \mathrm{j}$ & 225.29 & 152 & 10.24 \\
$5 \mathrm{k}$ & 100.55 & 100.6 & 10.59 \\
51 & 31.18 & 12.83 & 11.10 \\
$5 \mathrm{~m}$ & 41.23 & 9.06 & 10.98 \\
$5 \mathrm{n}$ & 44.41 & 75.67 & 10.94 \\
$5 \mathrm{o}$ & 22.01 & 17.32 & 11.25 \\
$5 \mathrm{p}$ & 7.58 & 5.59 & 11.71 \\
\hline
\end{tabular}

The active compounds $\mathbf{5 b}, \mathbf{e}, \mathbf{g}, \mathbf{l}, \mathbf{m}, \mathbf{o}$ and $\mathbf{p}$ were superimposed and merged into a single pharmacophore. Superimposed ligands are shown in Figure 3.

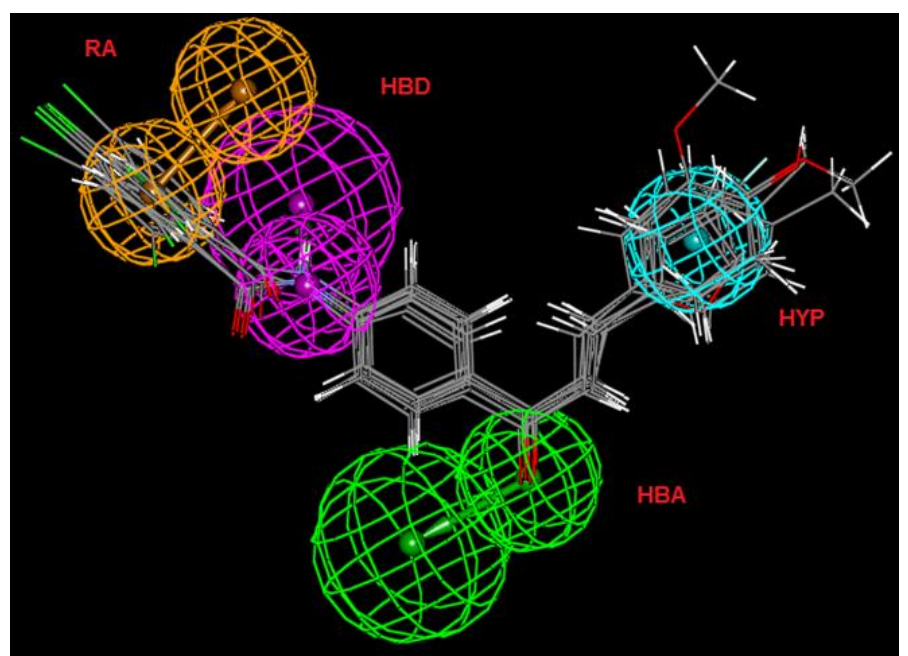

Fig. 3: The best generated pharmacophore hypothesis with the features considered hydrogen bond acceptor (HBA) colored in green, hydrogen bond donor (HBD) colored in magenta and aromatic ring (RA) colored in orange,

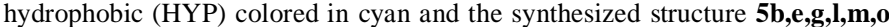
and $\mathbf{p}$ fitted in the pharmacophore.

\section{D QSAR Study \\ Development of QSAR Models}

QSAR analyses for antiproliferative activities of the prepared sixteen derivatives were established in order to correlate the observed activities with the structural features of the synthesized compounds. Thus, a total of 16 ligands with their measured pIC50s against PC3 cell line, were used to calculate the molecular properties by using the Calculate Molecular Properties protocol. Genetic function approximation (GFA) was applied to search for the best possible QSAR regression equation capable of correlating the variations in the biological activities of the training set compounds with variations in the generated descriptors. The 
linear Multiple Linear Regression (MLR) model was then constructed. The analysis was run by means of the DS 2.5 software (Discovery Studio 2.5, Accelrys, Co., Ltd., San Diego, CA, USA). Compounds $\mathbf{5 e}, \mathbf{5 i}$ and $\mathbf{5 p}$, one active, moderate and inactive, were used as an external test subset for validating the QSAR model. It was validated by the leave one-out cross-validation through setting the folds to a number much larger than the number of samples, $r^{2}$ (squared correlation coefficient value) and $r^{2}$ prediction (predictive squared correlation coefficient value), residuals between the predicted and experimental activity of the test set and training set.

\section{QSAR Study Results}

Equation (1). Represents the best performing QSAR model for the activity against prostate cancer cell line, PC3;

\section{$-\log$ IC50 $=3.6539+0.5232$ CHI_V_3_P +0.30038 Jurs_RNCS - 14.288 Shadow_YZfrac (1)}

According to equation (1), the QSAR model was represented graphically by scattering plots of the experimental versus the predicted bioactivity values $-\log \mathrm{IC} 50$ for the training set compounds as shown in Figure 4. The method used to build the model was Least-Squares, $r^{2}=0.886, r^{2}(\operatorname{adj})=0.851, r^{2}($ pred $)=$ 0.741 , Least-Squared error $=0.218$, where $r^{2}$ (adj) is $r^{2}$ adjusted for the number of terms in the model; $r^{2}$ (pred) is the prediction $c$, equivalent to $q^{2}$ from a leave-1-outcross validation.

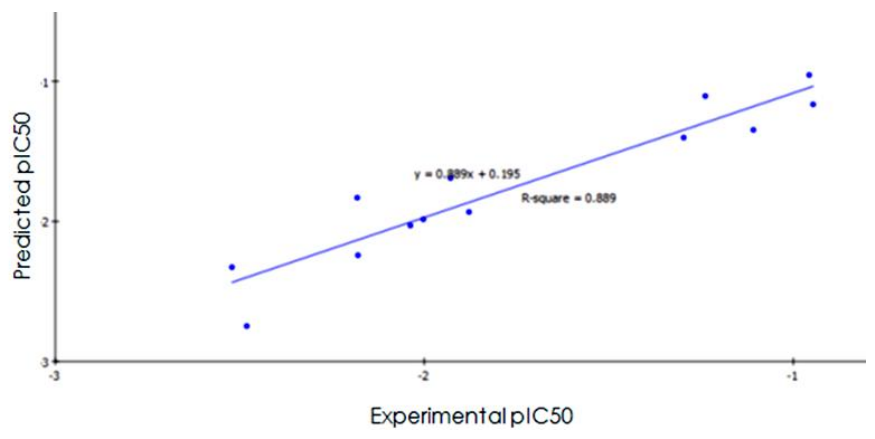

Fig. 4: Predicted versus experimental $\mathrm{pIC}_{50}$ of the tested compounds against $P C 3$ cell line according to equation $(\mathbf{1}), \mathrm{r}^{2}=0.889$.

In conclusion, equation (1) suggested that the antiprostate activity of the compounds was positively affected by the descriptors CHI_V_3_P and Jurs_RNCS, while Shadow_YZfrac contributed negatively to the antiproliferative activity of the compounds against PC3. The shadow indices are those geometric descriptors that characterize the shape of the molecules (Rohrbaugh and Jurs, 1987). CHI_V_3_P (molecular connectivity index) is one of the topological descriptors which are a certain class of descriptors that do not rely on a three-dimensional model. The calculations are derived from the 2D topology of a molecule. Combinations of several molecular connectivity indices, describe three categories of the molecular structure information; the degree of branching, the size and the degree of flexibility ( $\mathrm{Hu}$ et al., 2004). While, Jurs_RNCS, a spatial descriptor, encodes electronic properties of charges repartition on the molecule (Sattwa and Kunal 2009). Jurs descriptors are those ones that combine shape and electronic information that characterize molecules (Stanton and Jurs, 1990; Rohrbaugh and Jurs, 1987). Those surface area descriptors may indicate the factors influencing the binding of a ligand to a target.

\section{QSAR Validation}

The robustness of the QSAR model (1) was verified by using; Leave-one-out (LOO) internal validation $\left(r^{2}=0.886\right)$. Cross-validation was also employed where $q^{2}$, which is equivalent to $r^{2}$ (pred), was 0.741 . In addition, it was validated by measuring the residuals between the experimental and the predicted activities of the training set, Table 5 .

Table 5: Experimental activities of the synthesized derivatives against the predicted activities according to equations 1 .

\begin{tabular}{cccc}
\hline Cpd.No. & $\begin{array}{c}\text { Experimental } \\
\text { activity } \\
\text { pIC50 }\end{array}$ & $\begin{array}{c}\text { Predicted } \\
\text { activity } \\
\text { pIC50 }\end{array}$ & Residuals \\
\hline $\mathbf{5 a}$ & -2.17984 & -2.24178 & 0.0619385 \\
$\mathbf{5 b}$ & -0.946452 & -1.16476 & 0.218304 \\
$\mathbf{5 c}$ & -2.03782 & -2.02937 & -0.00844524 \\
$\mathbf{5 d}$ & -2.48116 & -2.74738 & 0.266215 \\
$\mathbf{5 g}$ & -2.52127 & -2.32713 & -0.194141 \\
$\mathbf{5 F}$ & -1.92906 & -1.69092 & -0.23814 \\
$\mathbf{5 h}$ & -2.18184 & -1.83118 & -0.350656 \\
$\mathbf{5 j}$ & -2.0026 & -1.98501 & -0.0175905 \\
$\mathbf{5 K}$ & -1.10823 & -1.34712 & 0.238893 \\
$\mathbf{5 l}$ & -0.957128 & -0.955122 & -0.00200613 \\
$\mathbf{5 m}$ & -1.87892 & -1.93298 & 0.0540609 \\
$\mathbf{5 n}$ & -1.23855 & -1.10519 & -0.133357 \\
$\mathbf{5 0}$ & -1.2971 & -1.40202 & 0.104925 \\
\hline
\end{tabular}

Moreover, the experimental and expected activities as well as the residuals of the compounds, used as statistical outliers in building the model, were presented in Table 6. The predicted activities by the generated QSAR model were close to those observed experimentally, this indicates that this model could be applied for further prediction of more effective hits having the same framework.

Table 6: Experimental activities of compounds $\mathbf{5 e}, \mathbf{5 i}$ and $\mathbf{5 p}$, used as statistical outliers against the predicted activities according to equations 1.

\begin{tabular}{cccc}
\hline Cpd.No. & $\begin{array}{c}\text { Experimental } \\
\text { activity pIC50 }\end{array}$ & $\begin{array}{c}\text { Predicted } \\
\text { activity pIC50 }\end{array}$ & Residuals \\
\hline $\mathbf{5 e}$ & -1.25959 & -0.933088 & -0.326502 \\
$\mathbf{5 i}$ & -2.99238 & -2.22641 & -0.765968 \\
$\mathbf{5 p}$ & -0.747412 & -1.83988 & 1.09247 \\
\hline
\end{tabular}

\section{Docking Studies}

Cathepsin B, a therapeutic target for the possible control of tumor progression, consequently, inhibitors of cathepsin B reduces both tumor cell mobility and in vasiveness in vitro (Frlan and Gobec 2006). In this work, the synthesis of a small library of chalcones and a study of their role as inhibitors of cathepsin B was carried out. First, the protocol of the docking was validated by 
reproducing the binding structure of the DPN ligand to active site of cathepsin B protein in the crystal complex (PDB code: 1GMY) (Greenspan et al., 2001), fig. 5.

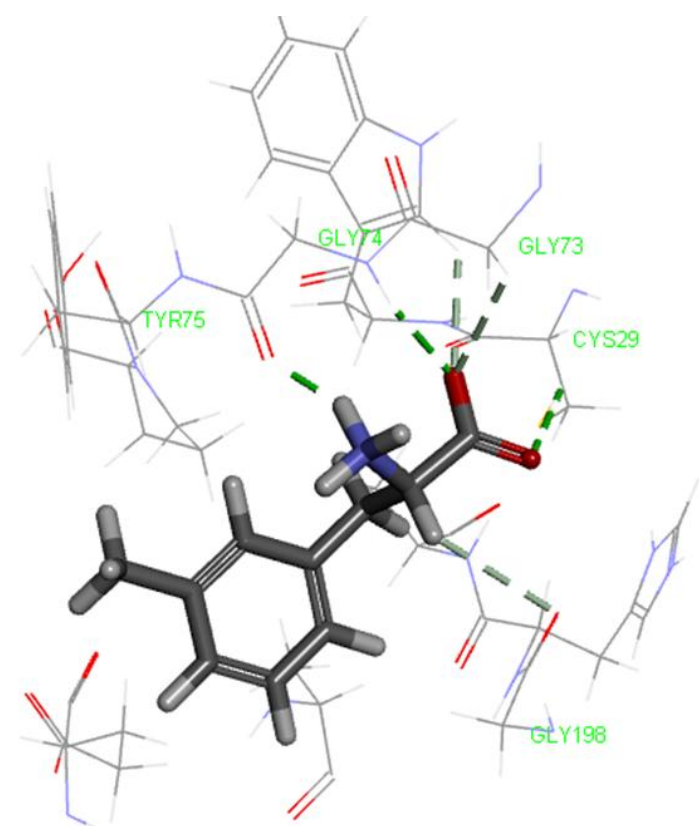

Fig. 5: The binding mode of DPN into the active site of CatB, PDB code $1 \mathrm{GMY}$, showing the important key binding interactions.

Molecular docking was performed using Accelrys Discovery studio 2.5 software using the Dock ligands (CDOCKER). Cathepsin B is a bilobal protein. The active site, in addition to the substrate-binding cleft, are both located at the interface between these two lobes. The peptide bond cleavage is catalyzed by a cysteine residue Cys 29 on the left lobe that interacts then with a histidine residue His 199 on the right lobe. The active site of cathepsin B is formed by an activated Cys, a His and Asn residues; near the active site there are two main pocket interactions, a large hydrophobic one and a smaller one, which is more solvent-accessible (Xulin et al., 2005). The binding pocket of the cathepsin $\mathrm{B}$ is defined by the following amino acid residues: Gln23, Cys29, Gly74, His110, His111, Val176, Leu181, Met196, Gly198, Trp221 and Glu245. Additionally, the 3D structure shows that the inhibitor dipeptidyl nitrile (DPN) binds to the active site, that is formed by amino acids Gln24, Cys27-Trp31, Gly69, Asp70, Asn73-Pro77, Ala174, Gly199-Ala201 and Glu245, close to the interface of the protein dimer. The inhibitor also forms a covalent sulfur bond with protein amino acid Cys30 (Galeazzia et al., 2003). In general, the design of cysteine proteinase inhibitors nearly follow the same strategy, where it depends on the presence of an electrophilic functionality that reacts with the thiolate moiety of the cysteine, Cys-29. The active site Cys-29 is then acylated by the inhibitor, which is followed by the transfer of acetyloxy moiety of the inhibitor catalyzed by His-199. Therefore, two vital amino acids, in the active site, are irreversibly blocked. Moreover, calculation of the total energy of stabilization of the enzyme- inhibitor complex proved that the dominant contribution is due to the strong electrostatic interaction between the ligand and the positively charged imidazolium rings of His110 and His111 (Gluza and Kafarski, 2011; Feng et al., 1996).

It could be concluded that the amino acid residue Cys 29 plays an important role in cathepsin B catalyzed mechanism (Xulin et al., 2005). Whereas, the cysteine thiol in the active site of the enzyme comprise thiolate characteristics which forms the covalent complex with the inhibitor, this leads to the loss of enzymatic activity (Otto and Schirmeister 1997 \& Turk et al., 2000). Whereas, cathepsin B is irreversibly inactivated by modification of the cysteine thiol through nucleophilic displacement or through conjugate addition to the electrophilic warhead of the inhibitors (Donkor 2000; Talanian et al., 2000).

In the present study, docking of the active derivatives, $\mathbf{5 b}$ and 5p, into the active site of the enzyme were performed, Fig. 6, 7. The results showed that the two compounds maintained the essential key interaction with Cys 29 through a hydrogen bond between the thiol and the carbonyl in the chalcone moiety. Whilst, compound 5p demonstrated a similar positioning and interaction mode to the inhibitor DPN, forming hydrogen bond interactions with Cys 29, Gly 73, Gly 198 in addition to Gly 74. While, compound $\mathbf{5 b}$ showed an interaction binding mode similar to that of the reversible and irreversible inhibitors, where the thiol group is acylated, this was further augmented by another hydrogen bond interaction with the imidazole ring of His 199. Other important hydrogen bonds were also formed between $\mathbf{5 b}$ and Gly 73, Gly 74, Tryp 30 and Gly 198. Moreover, CDOCKER interaction scores compared to DPN revealed interesting results that might justify the biological activity of the compounds as shown in Table 7.

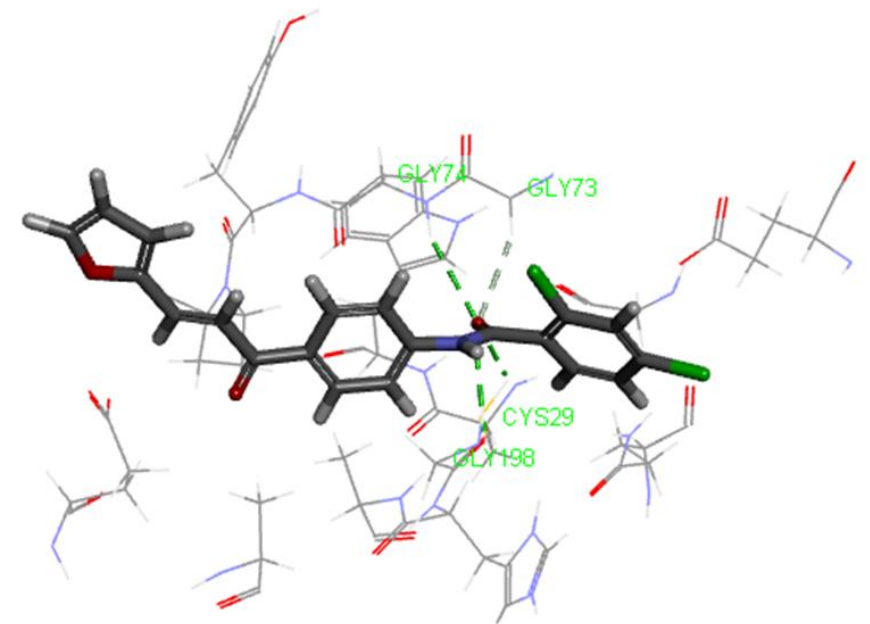

Fig. 6: The binding mode of $\mathbf{5 p}$ into the active site of CatB, PDB code 1GMY, showing the important key binding interactions.

Table 7: Docking scores of the tested compounds $5 \mathbf{b}, \mathbf{5 p}$ and DPN.

\begin{tabular}{ccc}
\hline Cpd.No. & $\begin{array}{c}\text { CDOCKER Energy } \\
\text { (Kcal/mol) }\end{array}$ & $\begin{array}{c}\text { CDOCKER Interaction } \\
\text { Energy (Kcal/mol) }\end{array}$ \\
\hline DPN & -38.565 & -28.953 \\
$5 \mathrm{~b}$ & -20.462 & -39.370 \\
$5 \mathrm{p}$ & -13.444 & -32.947 \\
\hline
\end{tabular}




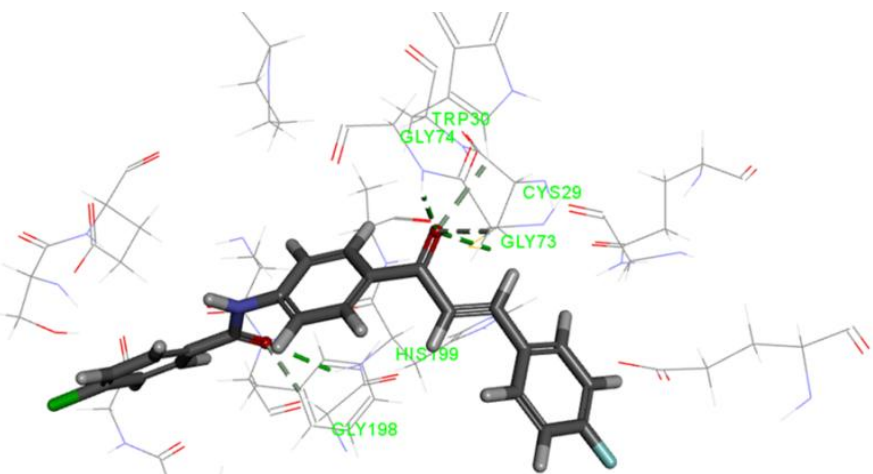

Fig. 7: The binding mode of $\mathbf{5 b}$ into the active site of CatB, PDB code 1GMY, showing the important key binding interactions.

\section{CONCLUSIONS}

In this study, synthesis of sixteen novel chalcone derivatives were established on the basis of spectral and microanalytical data, they were then evaluated for their antiprostate cancer activity. Compounds $\mathbf{5 b}, \mathbf{5 m}$ and $\mathbf{5 p}$ showed good activity both as antiproliferative and as inhibitors of Cathepsin B production. The developed QSAR model is statistically significant and has good predictive power as evidenced from the results of internal and external cross-validation parameters. Docking studies were also in agreement with the experimental results.

\section{Financial support and sponsorship: Nil.}

Conflict of Interests: There are no conflicts of interest.

\section{REFERENCES}

Aggarwal N and Sloane BF.Cathepsin B: Multiple roles in cancer, 2014; Proteomics Clin Appl, 2014; 8: 427-437.

Boumendjel A, Ronot X, Boutonnat J. Chalcones derivatives acting cell cycle blockers: Potential anticancer drugs? Current Drug Targets, 2009; 10:363-371.

Desmulle L, Bellahcene A, Dhooge W, Comhaire F, Roelens F, Huvaere K, Heyerick A, Castronovo V, Dekeukeleire D. Antiproliferative properties of prenylated flavonoids from hops (Humulus lupulus L.) in prostate cancer cell lines. Phytomedicine, 2006; 13:732-734.

Donkor IO. A survey of calpain inhibitors. Curr Med Chem, 2007; $11: 71$

Feng MH, Chan SL, Xiang Y, Huber CP, Lim C. The binding mode of an E-64 analog to the active site of cathepsin B.Protein Engineering, 1996; 9:977-86.

Fernandez P, Farre X, Nadal A, Fernandez E, Peiro N. Expression of cathepsins $\mathrm{B}$ and $\mathrm{S}$ in the progression of prostate carcinoma. Int J Cancer, 2001; 95:51-55.

Fidler IJ. Critical factors in the biology of human cancer metastasis. 28th G.H.A. Clowes Memorial Award Lecture. Cancer Res, 1990; 50:6130.

Frlan R, Gobec S. Inhibitors of cathepsin B. Curr Med Chem,2006; 13:2309-2327.

Fu Y, Hsieh TC, Guo J, Kunicki J, Lee MY, Darzynkiewicz $\mathrm{Z}, \mathrm{Wu} \mathrm{JM}$. Licochalcone-A, a novel flavonoid isolated from licorice root (Glycyrrhiza glabra) causes G2 and late-G1 arrests in androgenindependent PC3 prostate cancer cells. Biochem Biophys Res Comm,2004; 322:263-270.
Galeazzia R, Marucchinib C, Orenaa M, Zadra C. Stereoelectronic properties and activity of some imidazolinone herbicides: a computational approach. J Mol Str,2003; 640:191-200.

Gluza K, Kafarski P. Inhibitors of Proteinases as Potential AntiCancer Agents, Drug Development - A Case Study Based Insight into Modern Strategies, Dr. Chris Rundfeldt (Ed.), ISBN: 2011; 978-953- $307-$ 257-9, InTech, Available from: http://www.intechopen.com/books/drugdevelopment-a-case-study-basedinsight-into-modern-strategies/inhibitorsof-proteinases-as-potential-anti-cancer-agents.

Greenspan PD, Clark KL, Tommasi RA, Cowen SD, McQuire LW, Farley DL, van Duzer JH, Goldberg RL, Zhou H, Du Z, Fitt JJ, Coppa DE, Fang Z, Macchia W,Zhu L, Capparelli MP, Goldstein R, Wigg AM, Doughty JR, Bohacek RS, Knap AK. Identification of dipeptidyl nitriles as potent and selective inhibitors of cathepsin B through structure-based drug design. J Med Chem,2001; 44:4524-34.

Heidenreich A, Aus G, Bolla M, Joniau S, Matveev VB. EAU guidelines on prostate cancer. Eur Assoc Urol,2008; 53:68-80.

$\mathrm{Hu}$ QN, Liang YZ, Peng XL, Yin H, Fang KT. Structural interpretation of a topological index. 1. External factor variable connectivity index (EFVCI), J Chem Inform Comp Sci, 2004; 44:437-446.

Katsori AM, Hadjipavlou-Latina D. Chalcones in cancer: Understanding their role in terms of QSAR. Curr Med Chem,2009; 16:1062-1081.

Kim SH, Lee E, Baek KH, Kwon HB, Woo H, Lee ES, Kwon $\mathrm{Y}, \mathrm{Na}$ Y. Chalcones, inhibitors for topoisomerase I and cathepsin B and L, as potential anti-cancer agents. Bioorg Med Chem Lett, 2013; 23:3320-4.

Koblinski JE, Ahram M, Sloane BF. Unraveling the role of proteases in cancer. Clin Chim Acta,2000; 291:113-135.

Lee E, Jang I, Shin MJ, Cho H, Kim J, Kim HH, Cho KH. Chalcones as Novel Non-peptidic $\mu$-Calpain Inhibitors. Bull Kor Chem Soc, $2011 ; 32: 3459$.

Miyake H, Hara I, Eto H. Serum level of cathepsin B and its density in men with prostate cancer as novel markers of disease progression.Anticancer Res,2004; 24:2573-7.

Mohanam S, Jasti SL, Kondraganti SR, Chandrasekar N, Lakka SS, Kin Y, Fuller GN, Yung AW, Kyritsis AP, Dinh DH, Olivero WC, Gujrati M, Ali-Osman F, Rao JS. Down-regulation of cathepsin B expression impairs the invasive and tumorigenic potential of human glioblastoma cells. Oncogene2001; 20:3665-3673.

Nalla AK, GorantlaB, GondiCS, Lakka SS, RaoJS.Targeting MMP-9, uPAR, and cathepsin B inhibits invasion, migration and activates apoptosis in prostate cancer cells. Cancer Gene Therapy 2010; 17: 599613.

Nelson G, AlamAM, AtkinsonT, GurrapuS, KumarSJ. Synthesis and evaluation of $p-N, N$-dialkyl substituted chalcones as anticancer agents. Med Chem Res,2013; 22:4610-4614.

Nouh MA, Mohamed MM, El-Shinawi M, Shaalan MA, Cavallo-Medved D, Khaled HM, Sloane BF. Cathepsin B: a potential prognostic marker for inflammatory breast cancer.J Transl Med. 2011; 9 (1) $: 1$.

Olson OC and Joyce JA. Cysteine cathepsin proteases: regulators of cancer progression and therapeutic response. Nature Reviews Cancer, 2015; 15, 712-729.

Otto $\mathrm{H} \mathrm{H}$; Schirmeister T. Cysteine Proteases and Their Inhibitors. Chem Rev, 1997; 97:133-172.

Overmeyer JH, Young AM, Bhanot H, Maltese WA. A chalcone-related small molecule that induces methuosis, a novel form of non-apoptotic cell death, in glioblastoma cells. Molec Cancer, 2011; 10:69.

Rohrbaugh RH, Jurs PC. Descriptions of molecular shape applied in studies of structure/activity and structure/property relationships. Anal Chim Acta, 1987; 199:99-109.

Ruan H, Hao S, Young P, Zhang H. Targeting Cathepsin B for Cancer Therapies.Horiz. Cancer Res, 2015 2nd Quarter; 56: 23-40.

Ruan J, Zheng H, Rong X, ZhangJ, Fang W, Zhao P,Luo R. Over-expression of cathepsin B in hepatocellular carcinomas predicts poor prognosis of HCC patients. Molecular Cancer, 2016; 15:17. 
Sattwa A, Kunal M R. Predictive QSAR modeling of HIV reverse transcriptase inhibitor TIBO derivatives. Eur J Med Chem,2009; 44:1509-1524.

Shah A, Khan AM, Qureshi R, Ansari FL, Nazar MF, Syed SS. Redox behavior of anticancer chalcone on a glassy carbon electrode and evaluation of its interaction parameters with DNA. Int J Molec Sci,2008; 9:1424-1434.

Shweta G, Neera R. Inhibitory potential of some chalcones on cathepsins B, H and L RSC Adv2015; 5:72937-72949.

Sinha AA, JamuarMP, Wilson MJ, RozhinJ, Sloane BF. Plasma membrane association of cathepsin B in human prostate cancer. Biochemical and immunogold electron microscopic analysis.The Prostate, 2001; 49:172-184.

Skehan P, Storeng R, Scudiero D, Monks A, McMahon J, Vistica D, Warren JT, Bokesch H, Kenney S, Boyd MR. New colorimetric cytotoxicity assay for anticancer-drug screening. J Natl Cancer Inst, 1990; 82:1107-1112.

Stanton D T, Jurs PC. Development and use of charge partial surface area structural descriptorsin computer-assisted quantitative structure-property relationship studies. Anal Chem,1990; 62:2323-2329.

Sunduru N, Agarwal A, Katiyar SB, Goyal N, Gupta S, Chauhana PMS. Synthesis of 2,4,6-trisubstituted pyrimidine and triazine heterocycles as antileishmanial agent, Bioorg Med Chem,2006; 14:77067715 .

Syam S, Abdelwahab SI, Al-Mamary MA, Mohan S. Synthesis of Chalcones with Anticancer Activities. Molecules,2012; 17:6179-6195.

Szliszka E, Czuba ZP, Mazur B, Paradysz A, Krol W. Chalcones and dihydrochalcones augment TRAIL-mediated apoptosis in prostate cancer cells. Molecules,2010; 15:5336-53.

Talanian, R. V.; Brady, K. D.; Cryns, V. L: Caspases. Biological Function, Specificity, and Design of Inhibitors. J Med Chem,20004; 3:3351-3371.
Tanaka Y, Tanaka R, Kawabata T, Noguchi Y, Himeno M. Lysosomal cysteine protease, cathepsin $\mathrm{B}$, is targeted to lysosomes by the mannose 6-phosphate-independent pathway in rat hepatocytes: sitespecific phosphorylation in oligosaccharides of the proregion. $\mathrm{J}$ Biochem,2000; 128:39-48.

Turk B, Turk D, Turk V. Lysosomal cysteine proteases: more than scavengers Biochim Biophys Acta,2000; 1477 (1-2) :98-111.

Xulin P, Ninghua T, Guangzhi Z, Yumei Z, Ruirui J. Amentoflavone and its derivatives as novel natural inhibitors of human Cathepsin B. Bioorg Med Chem,2005; 13:5819-5825.

Yun JM, Kweon MH, Kwon H, Hwang JK, Mukhtar H. Induction of apoptosis and cell cycle arrest by a chalcone panduratin A isolated from Kaempferia pandurata in androgen-independent prostate cancer cells PC3 and DU145. Carcinogenesis,2006; 27:1454-1564.

Zhou J, Geng G, Batist G, Wu JH. Synthesis and potential antiprostate cancer activities of ionone-based chalcones. Bioorg Med Chem Lett, 2009; 19: 1183-1186.

\section{How to cite this article:}

Soliman DH, Farrag AM, Omran O. Design, Synthesis and InSilico Studies of Novel Chalcones as Anti-Prostate Cancer and Cathepsin B Inhibitors. J App Pharm Sci, 2017; 7 (07): 010-020. 